\title{
Estimating the prevalence of comorbid conditions and their effect on health care costs in patients with diabetes mellitus in Switzerland
}

This article was published in the following Dove Press journal:

Diabetes, Metabolic Syndrome and Obesity:Targets and Therapy

I October 2014

Number of times this article has been viewed

\author{
Carola A Huber' \\ Peter Diem² \\ Matthias Schwenkglenks ${ }^{3}$ \\ Roland Rapold' \\ Oliver Reich' \\ 'Department of Health Sciences, \\ Helsana Group, Zürich, Switzerland; \\ ${ }^{2}$ Department of Endocrinology, \\ Diabetes and Clinical Nutrition, \\ Inselspital, Bern University Hospital, \\ and University of Bern, Bern, \\ Switzerland; ${ }^{3}$ Institute of Social and \\ Preventive Medicine, University of \\ Zürich, Zürich, Switzerland
}

Background: Estimating the prevalence of comorbidities and their associated costs in patients with diabetes is fundamental to optimizing health care management. This study assesses the prevalence and health care costs of comorbid conditions among patients with diabetes compared with patients without diabetes. Distinguishing potentially diabetes- and nondiabetes-related comorbidities in patients with diabetes, we also determined the most frequent chronic conditions and estimated their effect on costs across different health care settings in Switzerland.

Methods: Using health care claims data from 2011, we calculated the prevalence and average health care costs of comorbidities among patients with and without diabetes in inpatient and outpatient settings. Patients with diabetes and comorbid conditions were identified using pharmacy-based cost groups. Generalized linear models with negative binomial distribution were used to analyze the effect of comorbidities on health care costs.

Results: A total of 932,612 persons, including 50,751 patients with diabetes, were enrolled. The most frequent potentially diabetes- and nondiabetes-related comorbidities in patients older than 64 years were cardiovascular diseases (91\%), rheumatologic conditions (55\%), and hyperlipidemia (53\%). The mean total health care costs for diabetes patients varied substantially by comorbidity status (US\$3,203-\$14,223). Patients with diabetes and more than two comorbidities incurred US\$10,584 higher total costs than patients without comorbidity. Costs were significantly higher in patients with diabetes and comorbid cardiovascular disease (US\$4,788), hyperlipidemia (US\$2,163), hyperacidity disorders (US\$8,753), and pain (US\$8,324) compared with in those without the given disease.

Conclusion: Comorbidities in patients with diabetes are highly prevalent and have substantial consequences for medical expenditures. Interestingly, hyperacidity disorders and pain were the most costly conditions. Our findings highlight the importance of developing strategies that meet the needs of patients with diabetes and comorbidities. Integrated diabetes care such as used in the Chronic Care Model may represent a useful strategy.

Keywords: diabetes, comorbidity, chronic diseases, costs

\section{Introduction}

Diabetes is a highly prevalent chronic disease with a substantial medical and economic effect on health care systems worldwide. ${ }^{1,2}$ Because of diabetes, but also as a result of the various complications of diabetes, the burden is increasing tremendously. Diabetes is an established risk factor for comorbid chronic conditions such as cardiovascular diseases, musculoskeletal diseases, and mental diseases. ${ }^{3-5}$ Diabetes comorbidities account for a substantial proportion of the medical expenditures in patients with diabetes. ${ }^{6-9}$ Patients with diabetes and macrovascular and microvascular complications have total health care costs almost double those of patients without complications. ${ }^{10}$
Correspondence: Carola A Huber Department of Health Sciences, Helsana Group, PO Box 808I Zürich, Switzerland $\mathrm{Tel}+4$ I 43340 634 I

Fax +4I 433400434

Email carola.huber@helsana.ch 
According to a US study from the American Diabetes Association, more than a quarter of health care expenditures in five of eight comorbid diseases were attributed to diabetes. ${ }^{11}$ So far, most studies have focused only on a small selection of diabetes-related comorbidities and/or have limited their estimates to a single health care setting, mainly to inpatient hospital settings. ${ }^{9,12,13}$ Prior research showed that even conditions not directly related to diabetes, such as pain and depression, are more prevalent in diabetes, and thus emphasize the need to take into account both diabetes-related and nondiabetes-related comorbidities., ${ }^{3,16}$ Furthermore, Struijs and colleagues indicated in their study that the various comorbid diseases have different effects on the type of health care use in patients with diabetes, and they stress the importance of considering multiple health care settings. ${ }^{14}$

To the best of our knowledge, there are no comprehensive data on diabetes comorbid chronic conditions and their effect on health care costs in Switzerland. Because previous results may not be transferable to countries with different populations and health care delivery systems, it is important to evaluate the current situation of comorbidities in Swiss patients with diabetes. Therefore, the aim of our study was to provide a national overview of a wide range of (comorbid) chronic conditions in patients with diabetes compared with in patients without diabetes, as well as the associated health care costs across various health care settings for both patient groups. Distinguishing potentially diabetes-related and nondiabetes-related comorbidities in patients with diabetes, we also identified the most frequent chronic conditions and estimated their effect on costs.

\section{Material and methods}

\section{Data source and study population}

Patient-level health care claims data from the leading health insurance group (Helsana Group) in Switzerland were used. Switzerland is a federal parliamentary republic consisting of 26 cantons (states). It had a population of 8 million in 2012. The prevalence of diabetes is about $5 \% .{ }^{17}$ The Swiss health care system has mandatory coverage and is consumerdriven; that is, consumers (about 70\%), less employers $(25 \%)$ or the government (about $5 \%$ ), mainly pay health care costs through insurance premiums and out-of-pocket expenditures. ${ }^{18}$ In addition, the insurance coverage has a mandatory cost-sharing scheme consisting of co-payments and deductibles for all Swiss residents. Co-payments are a charge of $10 \%$ of the annual health care costs that every insured person has to pay (limited to US dollars [US\$770/ Swiss Francs [CHF] 700 per year). Deductibles range from
US\$330-\$2,750 (CHF 300-2,500) per year and can be chosen by the insured persons. The standard deductible is US\$330 (CHF 300), but to reduce premiums, the insured persons can choose a higher deductible (US\$550, \$1,650, $\$ 2,200$, or $\$ 2,750$ ).

Helsana covers about 1.2 million Swiss residents with mandatory health insurance coverage in all 26 cantons. Available data comprise information on health care use, prescription drugs and laboratory tests, and the associated costs from all health care settings (eg, outpatient [primary and secondary care], inpatient, and nursing). These data achieve a high level of completeness, as the recorded insurance claims cover almost all health care and pharmacy invoices. We performed a cross-sectional study including all subjects who were at least 18 years old and were enrolled in 2011. According to national ethical and legal regulations, ethical approval was not needed for this study.

\section{Identification of patients with diabetes and comorbidities}

According to the Anatomical Therapeutic Chemical (ATC) classification system, all prescription drug items are coded and assigned to an ATC code in our database. ${ }^{19}$ Using the pharmacy-based cost group model, certain ATC codes can be assigned to different chronic diseases..$^{20}$ In the absence of clinical diagnoses, this mapping approach provides a direct measure of treated chronic diseases and is commonly used as a reliable method to identify chronically ill patients in administrative databases. ${ }^{21-23}$ In addition, we used a modified version of the pharmacy-based cost group model, which included an updated and improved approach to the classification of medications. Patients were identified as having diabetes mellitus when they were prescribed at least one oral blood glucose-lowering drug (ATC code A10B), insulin (ATC code A10A), or another drug used in diabetes (ATC code A10X). We included a total of 22 chronic diseases based on the mapping approach for all chronic conditions performed by Huber and colleagues. ${ }^{24}$ In addition, we differentiated the chronic conditions (without diabetes) according to Struijs and colleagues and Du and colleagues between potentially diabetes-related and nondiabetes-related comorbidities among patients with diabetes. ${ }^{14,16}$ Diabetes-related comorbidities included cardiovascular diseases such as hypertension, hyperlipidemia, and glaucoma. Nondiabetes-related comorbidities included hyperacidity-related disorders, bone diseases (osteoporosis), cancer, dementia, epilepsy, gout/ hyperuricemia, HIV, intestinal inflammatory diseases, iron deficiency anemia, migraines, pain, Parkinson's disease, 
psychological disorders, respiratory illness, rheumatologic conditions, thyroid disorders, and tuberculosis.

\section{Estimation of prevalence and health care costs}

We calculated the prevalence of each chronic disease by dividing the number of patients falling into the given disease group by the total number of persons included in the study for both patients with and patients without diabetes. Because chronic diseases are strongly associated with age, we report the prevalence for the age groups of 18-64 years and older than 64 years. Afterward, we compared the prevalence rates for both age groups by using chi-square tests. In a further step, we calculated the frequencies and proportions of the patient characteristics as well as the mean and median of total health care costs by comorbidity status among patients with and without diabetes. The comorbidity status was classified as no comorbidity, one comorbidity, two comorbidities, and more than two comorbidities among patients with diabetes. The (co)morbidity status was categorized with zero, one, two, or more than two chronic conditions among patients without diabetes. Differences between sex and age groups within the given comorbidity status groups were tested by chi-square tests. To compare the mean health care costs across the different comorbidity groups, we used Wilcoxon signed rank tests. Total health care costs were defined as the sum of payments made by the mandatory health insurance for outpatient and inpatient care per patient/year. Outpatient costs included payments for office-based physician visits (primary care physicians, specialists), hospital ambulatory visits, paramedical visits, nursing, laboratory tests, prescription drugs, and medical devices. Cost from the inpatient setting included payments for hospitalization, rehabilitation, nursing home, and emergency transport services, including all associated costs of medications, laboratory tests, and medical devices.

Multiple linear regression analyses were performed to determine the effect of comorbidities on health care costs among patients with and without diabetes. Given the skewed nature of the cost distribution, we used negative binomial regression models with a linear link function that provided absolute values in CHF as estimates. Afterward, the CHF values were converted to US\$ at the 2011 exchange rate of 1 CHF to 1.1 US\$. Overall, we fitted four cost models for patients with diabetes and four models for patients without diabetes, according to their comorbidity status. The reference group in the respective models included patients without comorbidity among persons with diabetes and those without any chronic condition among persons without diabetes. In addition, we estimated regression models to assess the effect of the ten most frequent potentially diabetes- and nondiabetes-related comorbidities on health care costs among patients with diabetes. All multivariate regression models included age, and sex, to correct for potential confounding. For the purposes of this study, a $P$-value of $\leq 0.05$ was considered statistically significant. Analyses were performed using R, version 2.14.2 (R Foundation for Statistical Computing, Vienna, Austria).

\section{Results}

A total of 932,612 persons including 50,751 patients with diabetes were included in the study sample. We distinguished the characteristics of patients with and without diabetes, showing overall a higher percentage of men $(54 \%)$ in the diabetes sample and a higher percentage of women in the nondiabetes sample (53\%; results not shown).

Table 1 shows the population characteristics and the prevalence of comorbidities among patients with diabetes compared with those in patients without diabetes for the respective age groups. Approximately $60 \%$ of the sample with diabetes was older than 64 years, whereas almost $75 \%$ of the sample without diabetes were younger than 65 years. The mean number of comorbid chronic conditions was 3.3 respectively 4.1 among patients with diabetes and significantly higher compared with patients without diabetes (1.2 respectively 3.0 conditions). The most frequent concurrent conditions in the population with diabetes were cardiovascular diseases including hypertension (18-64 years: 67\%; older than 64 years: $91 \%$ ), rheumatologic conditions (18-64 years: 54\%; older than 64 years: 55\%), pain (18-64 years: 45\%; older than 64 years: $50 \%$ ), and hyperlipidemia (18-64 years: $44 \%$; older than 64 years: $53 \%$ ).

Among patients without diabetes, the most commonly recorded comorbidities were cardiovascular diseases (older than 64 years: 63\%), rheumatologic conditions (18-64 years: $31 \%$; older than 64 years: $47 \%$ ), pain (18-64 years: $23 \%$; older than 64 years: $39 \%$ ), and psychological disorders (older than 64 years: $35 \%$ ). The majority of comorbidities occurred significantly more frequently in patients with diabetes than in patients without diabetes in both age groups. Among persons without diabetes, the proportion of patients with each chronic condition is significantly higher in the older age group compared with in the younger group. With the exception of epilepsy, intestinal inflammatory diseases, and psychosis, comorbid conditions occurred significantly more often in older patients with diabetes than in younger patients with diabetes. 
Table I Population characteristics and prevalence of comorbidities among patients with, compared with patients without, diabetes

\begin{tabular}{|c|c|c|c|c|c|c|}
\hline \multirow[t]{2}{*}{ Population characteristics } & \multicolumn{3}{|c|}{ Diabetes sample $(n=50,75 I)$} & \multicolumn{3}{|c|}{ Nondiabetes sample $(n=88 \mathrm{I}, 86 \mathrm{I})$} \\
\hline & $18-64$ years, $\%$ & $>64$ years, $\%$ & $P$-value & $18-64$ years, $\%$ & $>64$ years, $\%$ & $P$-value \\
\hline Total & 37.8 & 62.2 & & 74.3 & 25.7 & \\
\hline Sex & & & $<0.00 \mathrm{I}^{\dagger}$ & & & $<0.00 \mathrm{I}^{\dagger}$ \\
\hline Male & 59.1 & 51.4 & & 49.7 & 39.6 & \\
\hline Female & 40.9 & 48.6 & & 50.3 & 60.4 & \\
\hline $\begin{array}{l}\text { Mean number of chronic } \\
\text { conditions (standard deviation) }\end{array}$ & $3.3(2.0)$ & $4.1(1.9)$ & $<0.00 I^{\ddagger}$ & $1.2(1.6)$ & $3.0(2.1)$ & $<0.00 I^{\ddagger}$ \\
\hline \multicolumn{7}{|l|}{ Comorbidities $\pi$} \\
\hline Hyperacidity-related disorders & $34.9 * * *$ & $39.4 * * *$ & $<0.00 \mathrm{I}^{\dagger}$ & 14.4 & 30.8 & $<0.00 \mathrm{I}^{\dagger}$ \\
\hline Bone diseases (osteoporosis) & $1.4^{* * *}$ & $4.2^{* * *}$ & $<0.00 \mathrm{I}^{\dagger}$ & 0.7 & 7.0 & $<0.00 \mathrm{I}^{\dagger}$ \\
\hline Cancer & $1.7^{* * *}$ & 3.1 & $<0.00 \mathrm{I}^{\dagger}$ & 0.8 & 2.9 & $<0.00 \mathrm{I}^{\dagger}$ \\
\hline $\begin{array}{l}\text { Cardiovascular diseases } \\
\text { (including hypertension) }\end{array}$ & $67.1 * * *$ & $91.0 * * *$ & $<0.00 \mathrm{I}^{\dagger}$ & 13.2 & 62.8 & $<0.00 \mathrm{I}^{\dagger}$ \\
\hline Dementia & $1.4 * * *$ & 5.1 & $<0.00 \mathrm{I}^{\dagger}$ & 0.5 & 5.0 & $<0.00 \mathrm{I}^{\dagger}$ \\
\hline Epilepsy & $6.5^{* * * *}$ & $6.6 * * *$ & $0.467^{\dagger}$ & 2.2 & 4.2 & $<0.00 \mathrm{I}^{\dagger}$ \\
\hline Glaucoma & $3.9 * * *$ & $13.1^{* * *}$ & $<0.00 \mathrm{I}^{\dagger}$ & 0.9 & 10.2 & $<0.00 \mathrm{I}^{\dagger}$ \\
\hline Gout, hyperuricemia & $4.4^{* * *}$ & $9.3^{* * *}$ & $<0.00 \mathrm{I}^{\dagger}$ & 0.6 & 3.7 & $<0.00 \mathrm{I}^{\dagger}$ \\
\hline HIV & 0.3 & 0.1 & $<0.00 \mathrm{I}^{\dagger}$ & 0.3 & 0.01 & $<0.00 \mathrm{I}^{\dagger}$ \\
\hline Hyperlipidemia & $43.8^{* * *}$ & $53.4^{* * *}$ & $<0.00 \mathrm{I}^{\dagger}$ & 4.8 & 25.7 & $<0.00 \mathrm{I}^{\dagger}$ \\
\hline Intestinal inflammatory diseases & $0.6^{* * *}$ & 0.7 & $0.773^{\dagger}$ & 0.4 & 0.7 & $<0.00 \mathrm{I}^{\dagger}$ \\
\hline Iron deficiency anemia & $5.7^{* * *}$ & $6.4^{* * *}$ & $<0.00 \mathrm{I}^{\dagger}$ & 4.1 & 3.4 & $<0.00 \mathrm{I}^{\dagger}$ \\
\hline Migraine & $1.2^{*}$ & $0.3 * * *$ & $<0.00 \mathrm{I}^{\dagger}$ & 1.5 & 0.6 & $<0.00 \mathrm{I}^{\dagger}$ \\
\hline Pain & $45.3^{* * *}$ & $49.8^{* * *}$ & $<0.00 \mathrm{I}^{\dagger}$ & 22.6 & 39.0 & $<0.00 \mathrm{I}^{\dagger}$ \\
\hline Parkinson's disease & $1.4^{* * *}$ & $3.7^{* * *}$ & $<0.00 \mathrm{I}^{\dagger}$ & 0.4 & 3.0 & $<0.00 \mathrm{I}^{\dagger}$ \\
\hline $\begin{array}{l}\text { Psychological disorders } \\
\text { (sleep disorder, depression) }\end{array}$ & $32.3^{* * *}$ & $41.0 * * *$ & $<0.00 \mathrm{I}^{\dagger}$ & 15.0 & 35.4 & $<0.00 \mathrm{I}^{\dagger}$ \\
\hline Psychoses & $6.1^{* * *}$ & $6.3^{* * *}$ & $0.505^{\dagger}$ & 2.4 & 4.9 & $<0.00 \mathrm{I}^{\dagger}$ \\
\hline $\begin{array}{l}\text { Respiratory illness (asthma, chronic } \\
\text { obstructive pulmonary disease) }\end{array}$ & $11.8^{* * *}$ & $13.1 * * *$ & $<0.00 \mathrm{I}^{\dagger}$ & 5.9 & 10.5 & $<0.00 \mathrm{I}^{\dagger}$ \\
\hline Rheumatologic conditions & $54.3^{* * *}$ & $55.0^{* * *}$ & $0.107^{\dagger}$ & 30.8 & 47.1 & $<0.00 \mathrm{I}^{\dagger}$ \\
\hline Thyroid disorders & $7.2^{* * *}$ & $9.3^{* * *}$ & $<0.00 \mathrm{I}^{\dagger}$ & 2.3 & 7.0 & $<0.00 \mathrm{I}^{\dagger}$ \\
\hline Tuberculosis & $0.2^{* * *}$ & 0.1 & $0.026^{\dagger}$ & 0.1 & 0.1 & $<0.00 \mathrm{I}^{\dagger}$ \\
\hline
\end{tabular}

Notes: ${ }^{\dagger}$ Chi-square test comparing age groups within each sample with/without diabetes; ${ }^{\ddagger}$ Wilcoxon test comparing age groups in sample with/without diabetes. ${ }^{\top} \mathrm{Chi}$-square test comparing sample of same age groups across sample with diabetes and without diabetes, respectively; $* P$-value, $\leq 0.05 ; * * * P$-value, $\leq 0.00$ I.

The patient characteristics and health care costs categorized by diabetes and comorbidity status are presented in Tables 2 and 3. Of the 50,751 patients with diabetes, almost all persons had at least one concurrent condition (96\%). Furthermore, there were higher proportions of men in the diabetes sample. Among the patients without diabetes $(n=881,861)$, a total of $530,320(60 \%)$ had at least one chronic condition other than diabetes, with a higher percentage of women. The majority of the persons with one or two chronic conditions were younger than 64 years old; comorbid patients with more than two conditions were equally represented in both age groups. The mean total health care costs in 2011 varied substantially between the comorbidity status in patients with diabetes, ranging from US\$3,203 in patients with only diabetes to US\$14,223 in patients with more than two concurrent conditions. Annual costs of both the outpatient setting (US\$1,403 versus $\$ 5,577$ ) and the inpatient setting (US\$553 versus $\$ 4,764$ ), as well as the cost of prescription drugs (US\$857 versus $\$ 3,726$ ), were highest in patients with diabetes and at least three comorbidities. Overall, the total health care costs are significantly higher in patients with diabetes and comorbidities than in patients with diabetes only. Among persons without diabetes, the mean total health care costs were highest in multimorbid patients with more than two chronic conditions (US\$10,429 versus \$928). The costs varied across the different health care settings, from US $\$ 4,535$ in the outpatient setting and US $\$ 3,488$ in the inpatient setting for patients without diabetes and with at least three chronic diseases. After splitting the cost estimates into two age groups (18-64 years and older than 64 years), we could still observe higher average health care costs in all categories, with the exception of older patients 


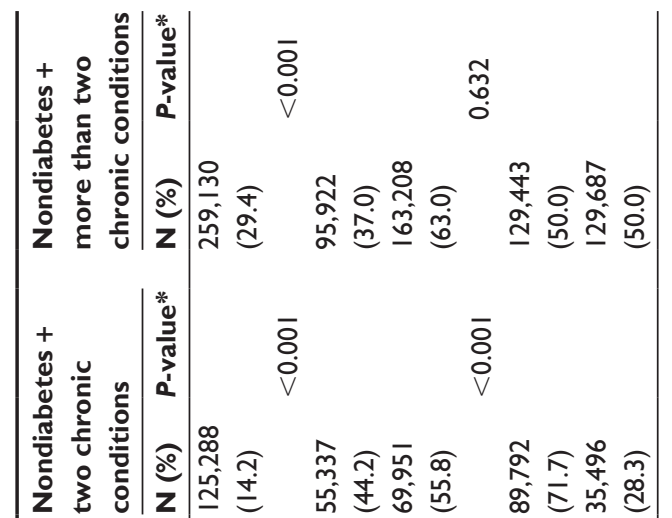

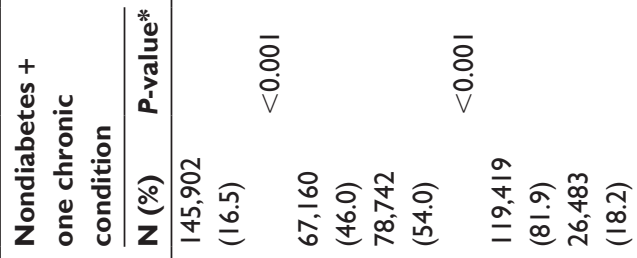

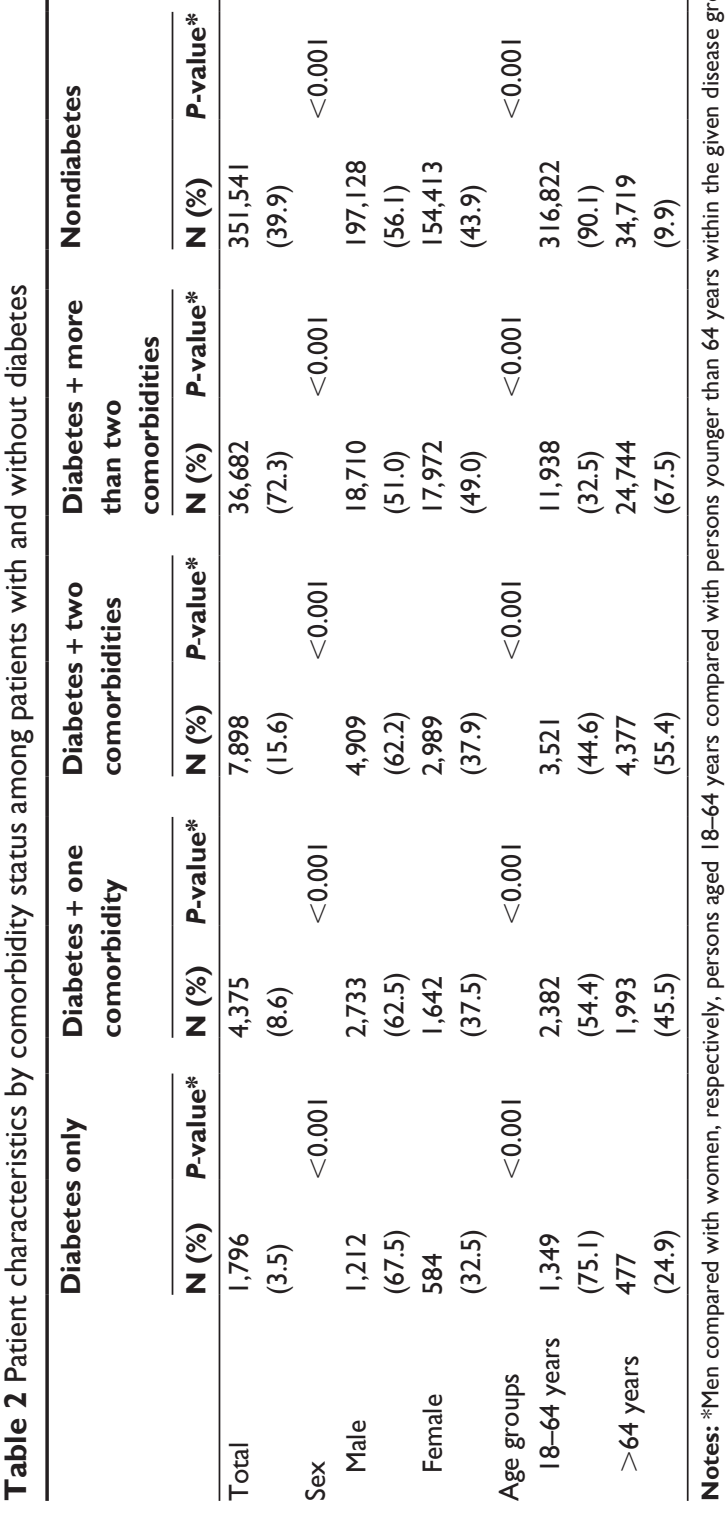

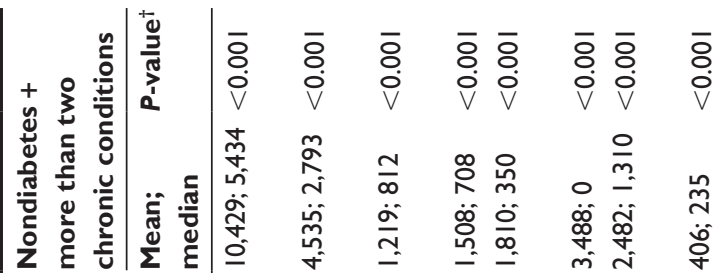

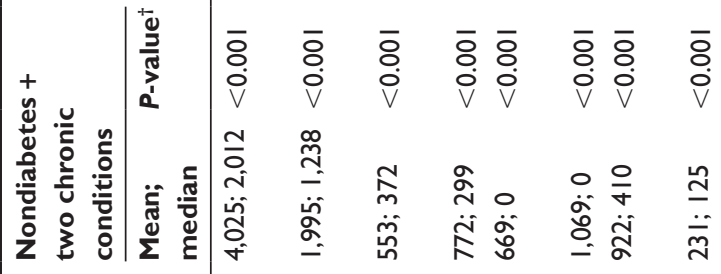

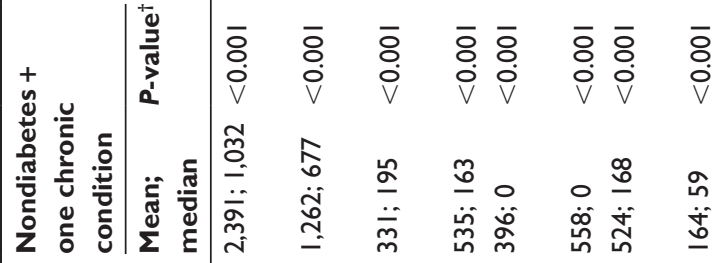

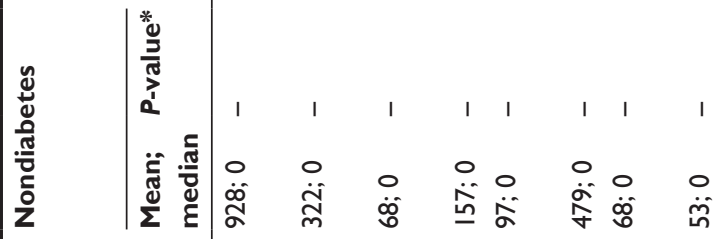

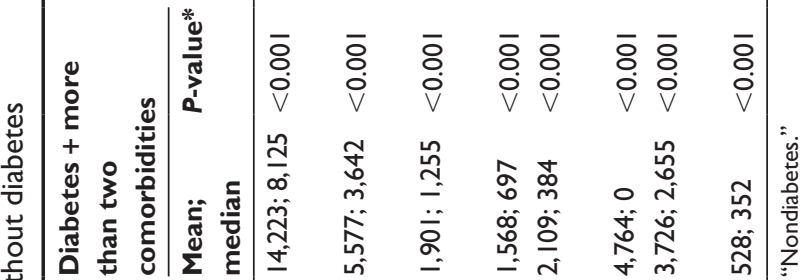
要

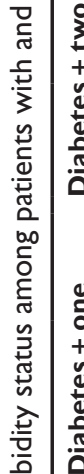

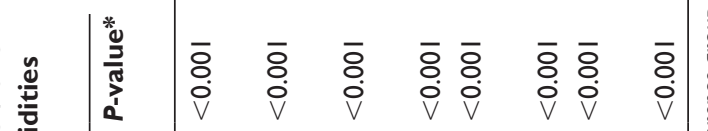

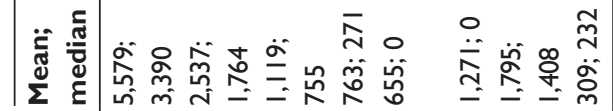

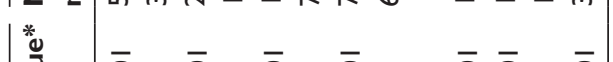

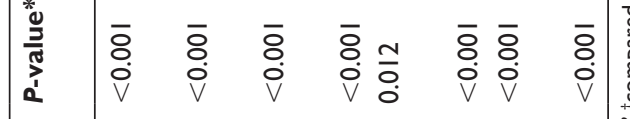

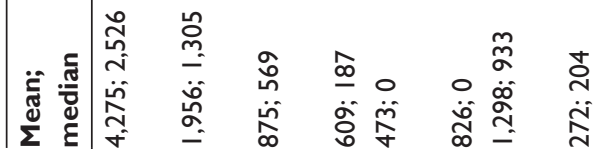
嗮

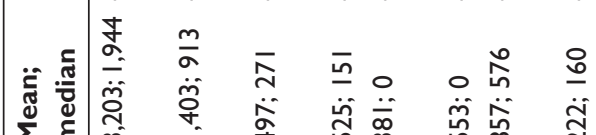

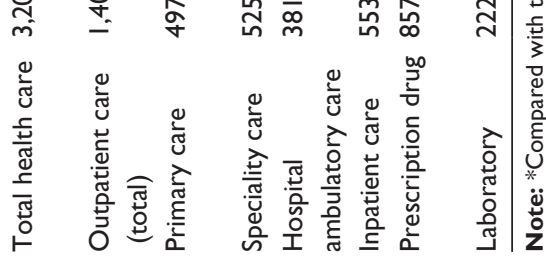


in the nondiabetes-only compared with the diabetes-only group (Figure 1).

Table 4 presents the results of the multivariate regression models estimating the effect of comorbidities on health care costs. After adjusting for sex and age, a substantial gradient between the number of comorbidities and health care costs in all health care settings could be observed among patients with and without diabetes. The total costs were almost US $\$ 10,600$ higher in patients with diabetes (coefficient, US\$10,584; $95 \%$ confidence interval [CI], US\$10,329-\$10,834) and about US\$8,300 higher in those without diabetes (coefficient, US\$8,293; 95\% CI, US\$8,199-\$8,389) with at least three chronic conditions compared with persons without comorbidity. The costs from both outpatient and inpatient settings, from prescription drugs and laboratory tests, each significantly increased with the number of concurrent (comorbid) chronic conditions in both samples.

The effect of the ten most common diabetes-related comorbidities on the health care costs is shown in Tables 5 and 6. All comorbidities caused a significant increase in health care costs in all considered settings. Regarding the diabetesrelated comorbidities of cardiovascular diseases and hyperlipidemia, the total health care costs were about US\$4,800 (coefficient, US\$4,788; 95\% CI, US\$4,576-\$5,000) and almost US\$2,200 (coefficient, US\$2,163; 95\% CI, US\$1,964-\$2,362) higher in these comorbid patients than in diabetes patients without the given chronic conditions. Furthermore, patients with diabetes and each comorbid pain-, psychological-, or hyperacidity-related disorder incurred
US $\$ 8,000$, on average, in higher total health care costs compared with the given reference group.

\section{Discussion}

In this study, we provide the first national overview of the diabetes comorbidity status in Switzerland, evaluating the prevalence and costs of potentially diabetes- and nondiabetesrelated comorbid conditions in inpatient and outpatient health care settings among patients with diabetes.

First, our study showed a higher proportion of adults older than 64 years among patients with diabetes than those without diabetes. This result suggests that our sample included a high number of patients with type 2 diabetes, which is typically associated with older ages. ${ }^{25}$ Furthermore, we identified cardiovascular diseases including hypertension, rheumatologic conditions, hyperlipidemia, and pain as the most common concurrent chronic conditions in patients with diabetes. These findings are in line with previous studies from Germany showing highest rates of hypertension, hyperlipidemia, and musculoskeletal diseases in patients with diabetes. ${ }^{16}$ From previous reports, cardiovascular diseases and hyperlipidemia are well known to be strongly associated with diabetes, even after adjustment for other risk factors of comorbidity such as age, sex, glycemia, and smoking. ${ }^{4}$ In addition, nondiabetesrelated comorbidities such as musculoskeletal and rheumatologic conditions could be observed as a concurrent chronic condition in a recent study. ${ }^{26}$ Longstanding diabetes may be harmful for knee and hip joints and includes a doubled risk for severe osteoarthritis needing arthroplasty. ${ }^{26}$ Overall, and

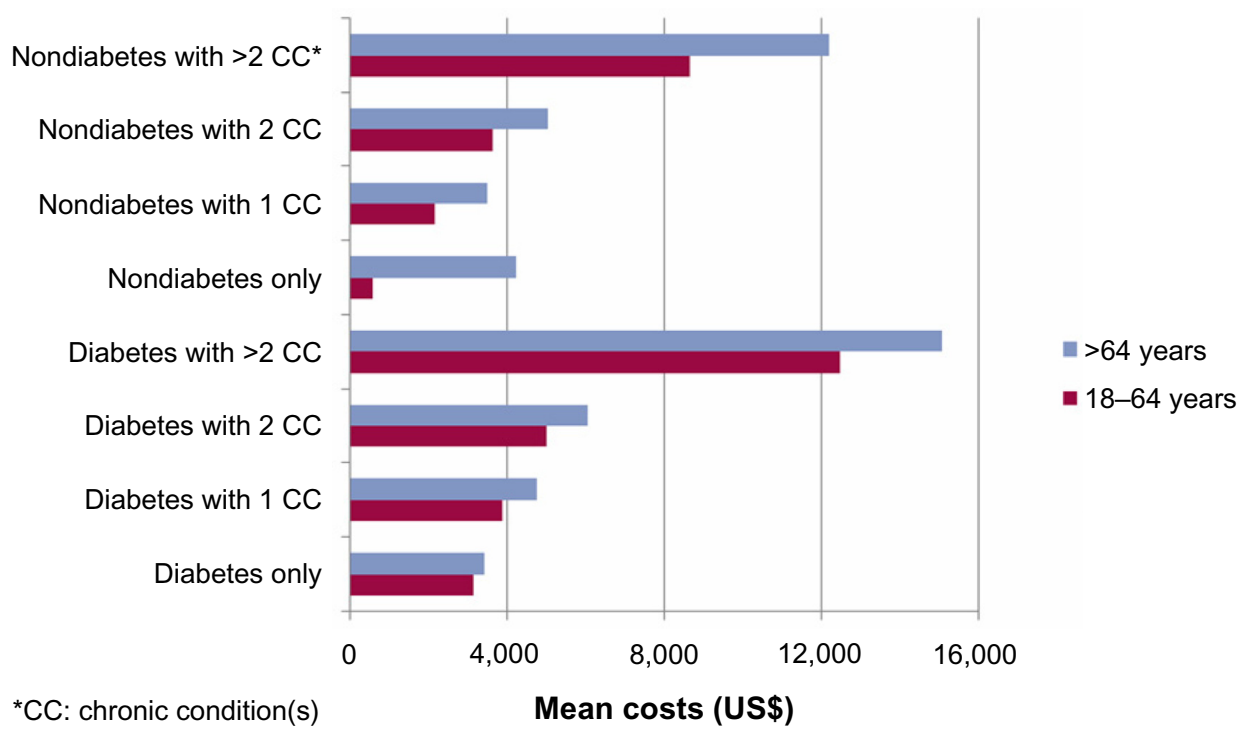

Figure I Mean total health care costs in patients with and without diabetes by comorbidity status. 


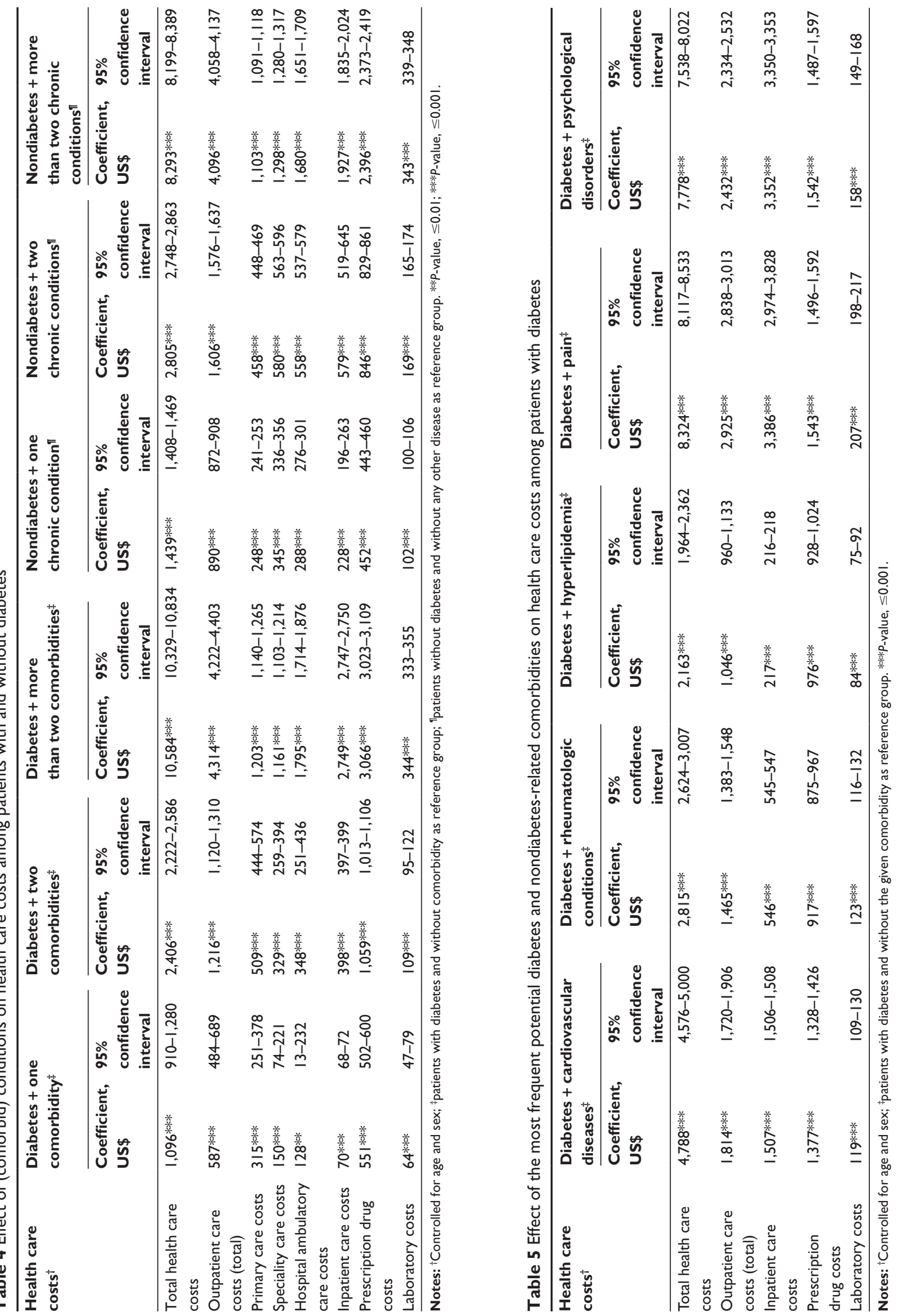




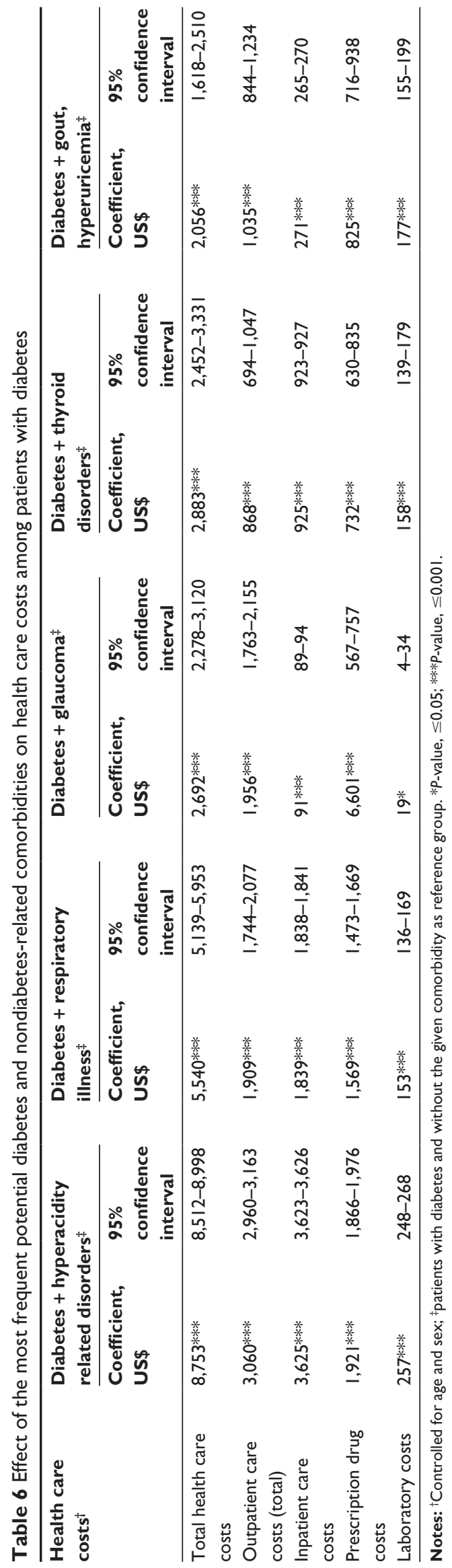

in line with numerous previous studies, our analyses showed that the majority of the comorbid chronic conditions occurred more frequently in patients with diabetes than in patients without diabetes. ${ }^{27-29}$

Furthermore, this study provides a description of the distribution of the total health care costs among patients with diabetes distinguished by with and without comorbidity. The costs varied substantially between the different groups categorized by comorbidity status. On average, the total annual costs in 2011 for diabetes care were about US $\$ 3,000(€ 2,000$; exchange rate of US\$1 to $€ 0.7$ in 2011) in patients with diabetes only and about US $\$ 14,000(€ 10,000)$ in patients with more than two concurrent conditions across all age groups. Overall, the total health care costs were significantly higher in patients with diabetes and comorbidities than in patients with diabetes only. Furthermore, the total health care costs were higher in patients with diabetes than in patients without diabetes in all comorbidity categories. However, we could observe one exception when we split the cost estimates into two age groups. Interestingly, there were higher average costs in the nondiabetes-only compared with the diabetes-only group among older patients. One explanation could be that in this age group, the distribution of costs was extremely skewed, with a mean of US\$4,200 (€3,000) and a median of US\$180 (€130). Because most of the previous studies included only a few major diabetes-related comorbidities, comparability of our direct average costs and those from other studies is limited. However, overall rising costs were related to an increased number of coexisting chronic diseases. For example, a study from Italy reported costs of $€ 1,040$ in patients with no major complications and of $€ 3,141$ in patients with two or more complications. ${ }^{13}$

Turning to the potential effect of comorbidities on health care expenditures, our results demonstrated that both potentially diabetes-related and nondiabetes-related comorbid conditions in patients with diabetes have a substantial effect on medical expenditures in health care systems. The increasing number of comorbid chronic conditions led to increasing annual medical expenditures, especially in total health care costs, outpatient costs, and prescription drug costs. Consistent with findings from previous studies, our analyses showed that suffering from at least two comorbidities has the highest effect on health care costs among patients with diabetes. ${ }^{12,13}$ Further studies also reported that concurrent conditions cause an increasing demand for total health care, a greater length of hospital stay, and higher total hospital charges, and that they lead to increasing mortality. ${ }^{12,14,30}$ Considering the ten most frequent concurrent chronic conditions, we 
observed varying effects, depending on the category of comorbidity. Nondiabetes-related comorbidities such as pain, hyperacidity-related disorders, and psychological disorders showed the strongest effect (up to a doubling) on total health care costs among patients with diabetes. This finding stresses the importance not only of directly diabetes-related comorbidities but also of nondiabetes-related comorbidities such as hyperacidity-related disorders. Wang and colleagues, for example, reported that about $40 \%$ of patients with diabetes suffer from gastroesophageal reflux disease. ${ }^{31}$ Moreover, the authors showed that patients with diabetes and neuropathy were more likely to suffer from this disease than patients without neuropathy. In addition, our results are in line with previous studies reporting a strong association between diabetes and pain, as well as psychological disorders inclusive of depression. ${ }^{32,33}$ Egede and colleagues found a strong association between depression in persons with diabetes and increased health care costs, regardless of age, sex, and other comorbidities. ${ }^{33}$

Interestingly, we could observe a greater effect of several comorbidities, such as cardiovascular disease or hyperlipidemia, on outpatient costs than on inpatient costs in patients with diabetes. One explanation may be that patients with diabetes and comorbidities are more likely to seek outpatient than inpatient care. ${ }^{34}$ Patients who suffer from chronic diseases, in particular, need continuous treatment and management in primary and outpatient specialist care. ${ }^{35,36}$ Our findings highlight the importance of continuing and strengthening diabetes management, addressing the needs for patients with multimorbidity in primary care. Beyond general guidelines for the care of patients, effective diabetes management requires an integrated, more holistic, and patient-centered concept. ${ }^{37}$ The Chronic Care Model (CCM) provides a useful approach for an improved and efficient management of multimorbid patients, mainly within primary care. The CCM was developed by Wagner and colleagues and represents an evidence-based conceptual framework for the treatment of chronically ill persons, including a comprehensive, multifaceted approach for patients with chronic diseases across the entire health care system. ${ }^{36,38,39}$ The six elements of the CCM include patient self-management support, evidencebased guidelines for decision support, delivery system design (eg, multidisciplinary teams), clinical information systems (eg, reminder systems), health care organization (eg, support of the leaders), and community resources. There is strong evidence that supports the CCM's effectiveness, showing an improvement in patient care and health outcomes such as glycated hemoglobin values and heart disease risk factors. ${ }^{40-42}$

This study has several strengths and limitations. One of the main strengths is that our study is the first that provides a national overview of a large number of both potentially diabetes- and nondiabetes-related comorbidities as well as their effect on health care costs across various health care settings in Switzerland. Furthermore, this study is based on a very comprehensive administrative claims database that encompasses a large population across Switzerland. Administrative data are a reliable, large-sized, and practicebased data source that provides sufficient information on morbidity and medical cost of patients. Moreover, these databases are very valuable when disease register or health survey studies are lacking.

However, this study also has several limitations. First, costs may be underestimated because in approximately $3 \%$ of all claims, invoices were not reimbursed by the health insurer and were paid out of pocket by the patient. Second, as medical diagnoses are not available in our data, we used drug-based diagnoses as a proxy for clinical diagnoses. Thus, comorbidities in patients with diabetes may be biased because not all ATC codes could be directly and uniquely assigned to the treatment of a certain disease. Furthermore, as population-based clinical parameters (eg, International Statistical Classification of Diseases and Related Health Problems, Tenth Revision) are very scarce in Switzerland, we could not compare our model performance with a diagnosedbased model. However, numerous studies could show that prescription data are a valid measure for prevalence of diabetes and other chronic diseases. ${ }^{20-23}$ For example, Cossman and colleagues showed that prescribed drug rates are a useful proxy for "disease-specific diagnoses prevalence." ${ }^{21}$ In addition, Chini and coworkers revealed drug data as a reliable source for prevalence estimates of chronic diseases. ${ }^{23}$ In particular, the use of prescription for antidiabetic drugs could be used for an accurate identification of diabetics across large populations. ${ }^{43,44}$ Third, several comorbidities, such as cardiovascular diseases including hypertension, were broadly defined, and further common complications in diabetes (eg, chronic kidney disease) could not be analyzed at all in the study because not every chronic disease allows an unambiguous identification by prescription drug items according to the used pharmacy-based cost group model. A fourth limitation of the study is that our estimates are not entirely representative of the general population. The sample included a slightly higher proportion of elderly persons than in the entire Swiss population. A detailed comparison of 
the proportions of the population characteristics between our study sample and the entire Swiss population has been described elsewhere. ${ }^{17}$

In conclusion, comorbidities in patients with diabetes are highly prevalent and have substantial consequences for medical expenditures. Interestingly, hyperacidity disorders and pain were the most costly conditions. Our findings highlight the importance of developing strategies that meet the needs of patients with diabetes and comorbidities. Furthermore, our study strengthens the demand for integrated diabetes care programs, which include multifaceted and patient-centered elements. Applying general principles of the CCM may contribute to more effective diabetes care in the context of the growing proportion of multimorbidity in the population.

\section{Disclosure}

Financial support for this study was provided by Merck, Sharp and Dohme and Co (MSD), Switzerland. CAH, RR, and OR received funding from MSD. PD received consulting fees from MSD. MS has no conflict of interest.

\section{References}

1. Boyle JP, Thompson TJ, Gregg EW, Barker LE, Williamson DF. Projection of the year 2050 burden of diabetes in the US adult population: dynamic modeling of incidence, mortality, and prediabetes prevalence. Popul Health Metr. 2010;8(1):29.

2. Zhang P, Zhang X, Brown J, et al. Global healthcare expenditure on diabetes for 2010 and 2030. Diabetes Res Clin Pract. 2010;87(3): 293-301.

3. Luijks H, Schermer T, Bor H, et al. Prevalence and incidence density rates of chronic comorbidity in type 2 diabetes patients: an exploratory cohort study. BMC Med. 2012;10(1):128.

4. Adler AI, Stratton IM, Neil HAW, et al. Association of systolic blood pressure with macrovascular and microvascular complications of type 2 diabetes (UKPDS 36): prospective observational study. $B M J$. 2000;321(7258):412-419.

5. Beckman JA, Creager MA, Libby P. Diabetes and atherosclerosis: epidemiology, pathophysiology, and management. JAMA. 2002;287(19):2570-2581.

6. Schmitt-Koopmann I, Schwenkglenks M, Spinas GA, Szucs TD. Direct medical costs of type 2 diabetes and its complications in Switzerland. Eur J Public Health. 2004;14(1):3-9.

7. Simpson SH, Corabian P, Jacobs P, Johnson JA. The cost of major comorbidity in people with diabetes mellitus. CMAJ. 2003;168(13):1661-1667.

8. O'Brien JA, Caro I, Getsios D, Caro JJ. Diabetes in Canada: direct medical costs of major macrovascular complications. Value Health. 2001;4(3):258-265.

9. Pagano E, Bo S, Petrinco M, Rosato R, Merletti F, Gregori D. Factors affecting hospitalization costs in Type 2 diabetic patients. $J$ Diabetes Complications. 2009;23(1):1-6.

10. Pelletier EM, Shim B, Ben-Joseph R, Caro JJ. Economic outcomes associated with microvascular complications of type 2 diabetes mellitus: results from a US claims data analysis. Pharmacoeconomics. 2009;27(6):479-490.

11. American Diabetes Association. Economic costs of diabetes in the US in 2012. Diabetes Care. 2013;36(4):1033-1046.
12. Lkhagva D, Kuwabara K, Matsuda S, Gao Y, Babazono A. Assessing the impact of diabetes-related comorbidities and care on the hospitalization costs for patients with diabetes mellitus in Japan. $J$ Diabetes Complications. 2012;26(2):129-136.

13. Morsanutto A, Berto P, Lopatriello S, et al. Major complications have an impact on total annual medical cost of diabetes: results of a database analysis. J Diabetes Complications. 2006;20(3):163-169.

14. Struijs JN, Baan CA, Schellevis FG, Westert GP, van den Bos GA. Comorbidity in patients with diabetes mellitus: impact on medical health care utilization. BMC Health Serv Res. 2006;6(1):84.

15. Bair MJ, Brizendine EJ, Ackermann RT, Shen C, Kroenke K, Marrero DG. Prevalence of pain and association with quality of life, depression and glycaemic control in patients with diabetes. Diabet Med. 2010;27(5):578-584.

16. Du Y, Heidemann C, Gößwald A, Schmich P, Scheidt-Nave C. Prevalence and comorbidity of diabetes mellitus among non-institutionalized older adults in Germany - results of the national telephone health interview survey 'German Health Update (GEDA)' 2009. BMC Public Health. 2013;13(1):166.

17. Huber CA, Schwenkglenks M, Rapold R, Reich O. Epidemiology and costs of diabetes mellitus in Switzerland: an analysis of health care claims data, 2006 and 2011. BMC Endocr Disord. 2014;14(1):44.

18. Herzlinger RE, Parsa-Parsi R. Consumer-driven health care: lessons from Switzerland. JAMA. 2004;292(10):1213-1220.

19. WHO Collaborating Centre for Drug Statistics Methodology. Guidelines for ATC Classification and DDD Assignment 2011. Oslo: WHO; 2010.

20. Lamers LM, van Vliet RC. The Pharmacy-based Cost Group model: validating and adjusting the classification of medications for chronic conditions to the Dutch situation. Health Policy. 2004;68(1):113-121.

21. Cossman RE, Cossman JS, James WL, et al. Correlating pharmaceutical data with a national health survey as a proxy for estimating rural population health. Popul Health Metr. 2010;8(1):25.

22. O'Shea M, Teeling M, Bennett $\mathrm{K}$. The prevalence and ingredient cost of chronic comorbidity in the Irish elderly population with medication treated type 2 diabetes: a retrospective cross-sectional study using a national pharmacy claims database. BMC Health Serv Res. 2013;13(1):23.

23. Chini F, Pezzotti P, Orzella L, Borgia P, Guasticchi G. Can we use the pharmacy data to estimate the prevalence of chronic conditions? a comparison of multiple data sources. BMC Public Health. 2011;11(1): 688.

24. Huber CA, Szucs TD, Rapold R, Reich O. Identifying patients with chronic conditions using pharmacy data in Switzerland: an updated mapping approach to the classification of medications. BMC Public Health. 2013;13(1):1030.

25. American Diabetes Association. Diagnosis and classification of diabetes mellitus. Diabetes Care. 2012;35(S1)(suppl 1):S64-S71.

26. Schett G, Kleyer A, Perricone C, et al. Diabetes is an independent predictor for severe osteoarthritis: results from a longitudinal cohort study. Diabetes Care. 2013;36(2):403-409.

27. Bytzer P, Talley NJ, Leemon M, Young LJ, Jones MP, Horowitz M. Prevalence of gastrointestinal symptoms associated with diabetes mellitus: a population-based survey of 15,000 adults. Arch Intern Med. 2001;161(16):1989-1996.

28. Mo F, Pogany LM, Li FC, Morrison H. Prevalence of diabetes and cardiovascular comorbidity in the Canadian Community Health Survey 2002-2003. ScientificWorldJournal. 2006;6:96-105.

29. Nichols GA, Hillier TA, Erbey JR, Brown JB. Congestive heart failure in type 2 diabetes: prevalence, incidence, and risk factors. Diabetes Care. 2001;24(9):1614-1619.

30. Lix LM, Quail J, Fadahunsi O, Teare GF. Predictive performance of comorbidity measures in administrative databases for diabetes cohorts. BMC Health Serv Res. 2013;13(1):340.

31. Wang X, Pitchumoni CS, Chandrarana K, Shah N. Increased prevalence of symptoms of gastroesophageal reflux diseases in type 2 diabetics with neuropathy. World J Gastroenterol. 2008;14(5):709-712. 
32. Krein SL, Heisler M, Piette JD, Makki F, Kerr EA. The effect of chronic pain on diabetes patients' self-management. Diabetes Care. 2005;28(1):65-70.

33. Egede LE, Zheng D, Simpson K. Comorbid depression is associated with increased health care use and expenditures in individuals with diabetes. Diabetes Care. 2002;25(3):464-470.

34. Green LA, Fryer GE Jr, Yawn BP, Lanier D, Dovey SM. The ecology of medical care revisited. N Engl J Med. 2001;344(26):2021-2025.

35. Wagner EH, Austin BT, Von Korff M. Organizing care for patients with chronic illness. Milbank Q. 1996;74(4):511-544.

36. Bodenheimer T, Wagner EH, Grumbach K. Improving primary care for patients with chronic illness. JAMA. 2002;288(14):1775-1779.

37. Piette JD, Kerr EA. The impact of comorbid chronic conditions on diabetes care. Diabetes Care. 2006;29(3):725-731.

38. Wagner EH, Austin BT, Von Korff M. Organizing care for patients with chronic illness. Milbank Q. 1996;74(4):511-544.

39. Bodenheimer T, Wagner EH, Grumbach K. Improving primary care for patients with chronic illness: the chronic care model, Part 2. JAMA. 2002;288(15):1909-1914.
40. Coleman K, Austin BT, Brach C, Wagner EH. Evidence on the Chronic Care Model in the new millennium. Health Aff (Millwood). 2009;28(1):75-85.

41. Nutting PA, Dickinson WP, Dickinson LM, et al. Use of chronic care model elements is associated with higher-quality care for diabetes. Ann Fam Med. 2007;5(1):14-20.

42. Vargas RB, Mangione CM, Asch S, et al. Can a chronic care model collaborative reduce heart disease risk in patients with diabetes? J Gen Intern Med. 2007;22(2):215-222.

43. Harris SB, Glazier RH, Tompkins JW, et al. Investigating concordance in diabetes diagnosis between primary care charts (electronic medical records) and health administrative data: a retrospective cohort study. BMC Health Serv Res. 2010;10(1):347.

44. Amed S, Vanderloo SE, Metzger D, et al. Validation of diabetes case definitions using administrative claims data. Diabet Med. 2011;28(4):424-427.

\section{Publish your work in this journal}

Diabetes, Metabolic Syndrome and Obesity: Targets and Therapy is an international, peer-reviewed open-access journal committed to the rapid publication of the latest laboratory and clinical findings in the fields of diabetes, metabolic syndrome and obesity research. Original research, review, case reports, hypothesis formation, expert opinion and commentaries are all considered for publication. The manuscript management system is completely online and includes a very quick and fair peer-review system, which is all easy to use. Visit http://www.dovepress.com/testimonials.php to read real quotes from published authors.

Submit your manuscript here: http://www.dovepress.com/diabetes-metabolic-syndrome-and-obesity-targets-and-therapy-journal 